\title{
HUSSERLIAN EIDETIC VARIATION AND OBJECTUAL UNDERSTANDING AS A BASIS FOR AN EPISTEMOLOGY OF ESSENCE
}

\author{
Robert MICHELS
}

\begin{abstract}
Vaidya has recently argued that while Husserl's method for acquiring knowledge of essence through use of our imagination is subject to a vicious epistemic circle, we can still use the method to successfully attain objectual understanding of essence. In this paper, I argue that the Husserlian objectual understanding-based epistemology envisaged by Vaidya suffers from a similar epistemic circularity as its knowledge-based foil. I argue that there is a straight-forward solution to this problem, but then raise three serious problems for an amended version of Vaidya's proposal and any similar Husserlian epistemology of essence. The paper closes with some general reflections on applying the Husserlian method to the contemporary notion of essence and on the idea of refocusing the epistemology of essence on understanding instead of knowledge.
\end{abstract}

KEYWORDS: epistemology of essence, eidetic variation, understanding, essence,

Husserl, Vaidya

\section{Essence and the Husserlian Approach to the Epistemology of Essence}

The notion of essence has recently seen a renaissance in philosophy. Essentialism has first come to prominence again in the context of the discussion about metaphysical modality which followed important advances in quantified modal logic in the 1970s and 1980s. The standard view emerging from the early debate simply identifies essentiality and metaphysical necessity, so that the essential properties of an object are just those for which it is metaphysically necessary that the object has them, if it exists. ${ }^{1}$ More recently, philosophers such as Fine, Correia,

1 See e.g. Saul Kripke, Naming and Necessity (Cambridge: Harvard University Press, 1980), Penelope Mackie, How Things Might Have Been (Oxford: Clarendon Press, 2006), David Wiggins, "The De Re 'Must': a Note on the Logical Form of Essentialist Claims," in Truth and Meaning, eds. Gareth Evans and John McDowell (Oxford: Oxford University Press, 1976), 285312.

(C) LOGOS \& EPISTEME, XI, 3 (2020): 333-353 


\section{Robert Michels}

and Lowe ${ }^{2}$ have embraced the notion of essentiality, but rejected the idea that it is identical or even definable in terms of metaphysical necessity. This development immediately raises questions about the epistemology of essence. While there are several well-developed approaches to the epistemology of metaphysical modality, such as the conceivability-based approach, ${ }^{3}$ it is not clear in how far we can depend on these approaches in order to explain knowledge of essence once we have distinguished this notion from that of metaphysical necessity. The epistemology of essence still offers many avenues for research. ${ }^{4}$

A historically important approach to the epistemology of essence which was specifically developed with this notion rather than the notion of metaphysical necessity in mind, is due to Husserl. It is the method of eidetic seeing or essential seeing ('Wesenserschauung'). Husserl's main idea is that knowledge of essence can be gained through a specific kind of intuition, the mentioned eidetic seeing, which we arrive at by starting from what Husserl calls intuition of something individual, ${ }^{5}$ which is an experience of a particular object. At the core of the method is a process he calls free variation. Roughly, this process is supposed to

${ }^{2}$ See Kit Fine, "Essence and Modality," Philosophical Perspectives 8 (1994): 1-16, Kit Fine, "Senses of Essence," in Modality, Morality, and Belief. Essays in Honor of Ruth Barcan Marcus, ed. Walter Sinnott-Armstrong (Cambridge: Cambridge University Press, 1995): 53-73, Fabrice Correia, "Generic Essence, Objectual Essence, and Modality," Noûs 40, 4 (2006): 753-67, Fabrice Correia, "On the Reduction of Necessity to Essence," Philosophy and Phenomenological Research 84, 3 (2012): 639-53, and E. J. Lowe, "Two Notions of Being: Entity and Essence," Royal Institute of Philosophy Supplements 62 (2008): 23-48.

3 See e.g. Stephen Yablo, "Is conceivability a guide to possibility?" Philosophy and Phenomenological Research 53, 1 (1993): 1-42, David J. Chalmers, "Does Conceivability Entail Possibility?" in Conceivability and Possibility, eds. Tamar Szabó Gendler and John Hawthorne (Oxford: Oxford University Press, 2002), 145-200. For an overview of this and other approaches, see Anand Vaidya, "The Epistemology of Modality," in The Stanford Encyclopedia of Philosophy, ed. Edward N. Zalta (The Metaphysics Research Lab. CSLI Stanford, 2017). https://plato.stanford.edu/archives/win2017/entries/modality-epistemology/.

${ }^{4}$ Contemporary works in the epistemology of essence include Bob Hale, Necessary Beings: An Essay on Ontology, Modality, and the Relations Between Them (Oxford: Oxford University Press, 2013), ch. 11 and Tuomas Tahko. "The Epistemology of Essence," in Ontology, Modality, Mind: Themes from the Metaphysics of E. J. Lowe, eds. Alexander Carruth and S. C. Gibb and John Heil (Oxford: Oxford University Press), 93-110, Tuomas E. Tahko, "Empirically-Informed Modal Rationalism," in Modal Epistemology After Rationalism, eds. Robert William Fischer and Felipe Leon (Cham: Synthese Library, 2017): 29-45, which build on E. J. Lowe, "What is the Source of Our Knowledge of Modal Truths?" Mind 121, 484 (2012): 919-950.

${ }_{5}^{5}$ Edmund Husserl, Ideas Pertaining to a Pure Phenomenology and to a Phenomenological Philosophy. First book. General Introduction to a Pure Phenomenology (The Hague: Martinus Nijhoff Publishers, 1983): 8. Original German term: 'individuelle Anschauung.' 
Husserlian Eidetic Variation and Objectual Understanding...

work as follows. ${ }^{6}$ First, we have an individual intuition of something which we then take as an example based on which we produce variations of it through our imagination. These variations have to fulfil two conditions. First, they have to be 'free,' in the sense that they may randomly deviate from the original experience or intuition, as long as they are still 'concretely similar'7 to it. Second, the number and sequence of variations also needs to be arbitrary, the idea being that it should not matter whether the process of imagining variations is aborted at a particular point or whether it could, at least theoretically, be extended infinitely. ${ }^{8}$ The purpose of the process is to isolate that which is invariant through all variations. This 'invariable ${ } h a t^{9}$ is then the essence of the experienced or imagined kind of object, that "without which the object cannot be intuitively imagined as such."10

There is of course much more to be said about this method considered in its historical context and in relation to Husserl's own conception of essence. ${ }^{11}$ The focus of this paper however is not on exegetical questions, but rather on a different question which we might put as follows: Can a variant of Husserl's method of essential seeing serve as the basis of an epistemology of essence, assuming the contemporary understanding of the latter notion developed by Fine, Correia, Lowe, and others? This question has been taken up in a recent paper by Vaidya. ${ }^{12}$ My main aim in this paper is to assess (and ultimately criticise) Vaidya's answer to a particular variant of the question which focuses on understanding instead of knowledge as its epistemological target notion.

Vaidya answers the question in the negative for the standard, knowledgebased approach to the epistemology of essence. In this paper, I will take a condensed presentation of Vaidya's argument for this conclusion as my starting point (section 2). In addition to this negative conclusion, Vaidya argues that our

${ }^{6}$ Husserl describes the process in detail in $\S 87$ "The method of essential seeing" in Edmund Husserl, Experience and Judgment. Investigations in a Genealogy of Logic, ed. Ludwig Landgrebe (London: Routledge \& Kegan Paul, 1973): 340-348. See also section II of Daniele De Santis, "Phenomenological Kaleidoscope: Remarks on the Husserlian Method of Eidetic Variation," in The New Yearbook for Phenomenology and Phenomenological Philosophy XI, eds. Burt Hopkins and John Drummond (London: Routledge, 2011): 20-23.

${ }^{7}$ Husserl, Experience and Judgment, 341.

${ }^{8}$ Husserl, Experience and Judgment, 342.

${ }^{9}$ Husserl, Experience and Judgment, 341.

${ }^{10}$ Ibid.

${ }^{11}$ See in particular Husserl's unpublished notes on eidetic variation from his Nachlass collected in Edmund Husserl, Zur Lehre vom Wesen und zur Methode der eidetischen Variation. Texte aus dem Nachlass (1891-1935). Husserliana Vol. XLI (Dordrecht: Springer, 2012).

12 Anand Jayprakash Vaidya. "Understanding and Essence," Philosophia 38, 4 (2010): 811-33. 


\section{Robert Michels}

main question can be answered positively, if we refocus the epistemology of modality on a different state, namely that of understanding. This second, positive argument from Vaidya's paper is presented in section 3, but then criticized in section 4. My main criticism will be that his positive proposal falls victim to an epistemic circularity very similar to the one which Vaidya identified in his critique of the knowledge-based variant of a Husserlian epistemology of essence. I will consider a response to this criticism and then present three arguments against the resulting approach and similar Husserlian approaches in section 5. In section 6, I close with a discussion of the prospects for other applications of Husserl's method in the contemporary epistemology of essence and for Vaidya's idea of refocusing the debate on understanding instead of knowledge.

\section{Vaidya's Negative Argument}

Vaidya argues that Husserl's method of free variation, which he calls Variation-inImagination, 'VIM' for short, cannot produce knowledge of essence. ${ }^{13}$ Vaidya's argument for the latter claim is based on three necessary conditions for attaining knowledge of essence. The first is Necessity-of-object-preservation, NOP:

NOP In order for an imaginative process, such as VIM, to yield a judgment about whether $P$ is an essential property of $o$ through property variation on $o$ via the construction of a set of scenarios $S_{1} \ldots S_{n}$, it must be the case that $o$ is preserved in the transition from each $S_{i}$ to $S_{k .}{ }^{14}$

In other words, for VIM to successfully produce in us knowledge of the essence of $o$, the scenarios, or in Husserl's term, variations, which we run through,

\footnotetext{
${ }^{13}$ See Vaidya, "Understanding and Essence," 820.

14 Vaidya, "Understanding and Essence," 821. Note that in accepting this condition as a constraint on VIM, Vaidya departs from Husserl, who insists that "variation [in imagination] depends precisely on this: that we drop the identity of the individual and change it imaginatively into another possible individual" (Husserl, Experience and Jusgment, 347-8). That Husserl's notion of essence admits of no essential truths (in the sense of eidetic law statements) about particular individuals is also explained in Rochus Sowa, "Essences and Eidetic Laws in Edmund Husserl's Descriptive Eidetics," The New Yearbook for Phenomenology and Phenomenological Philosophy VII, eds. Burt Hopkins and Steven Crowell (London: Routledge, 2007): 77-108. Note however that Vaidya's acceptance of NOP matches the predominant focus on individual essence which one e.g. finds in earlier contributions to the contemporary discussion of essence, such as Fine, "Essence and Modality" and Fine, "Senses of Essence." Later works broadened the focus by including a notion of generic essence (see e.g. Fabrice Correia, "Generic Essence, Objectual Essence, and Modality," Noûs 40, 4 (2006): 753-67, Kit Fine, "Unified Foundations for Essence and Ground," Journal of the American Philosophical Association 1, 2 (2015): 296-311), which is however also distinct from Husserl's notion.
} 
Husserlian Eidetic Variation and Objectual Understanding...

have to be such that the object $o$ stays the same. It is only the properties of the object which may be varied when passing from one such scenario to another; the object itself cannot be.

The second necessary condition for attaining knowledge of essence through VIM is:

NAC If a VIM yields knowledge of the essence of $o$ for a subject $A$, then it cannot be the case that the preservation of $o$ across $S_{1 \ldots} . . S_{n}$ is accidental. ${ }^{15}$

That the objects $o$ is preserved through $S_{1} \ldots S_{n}$ just means that $o$ has to be the same object throughout $S_{1} \ldots S_{n}$. In other words, NAC tells us that for an instance of VIM to yield knowledge of essence, it has to non-accidentally confirm to NOP. This second assumption is modelled on a general assumption about knowledge which is defended by several contemporary epistemologists, namely that it is not merely a matter of (a problematic kind of) epistemic luck that the subject has gained the relevant true belief. ${ }^{16}$ The idea captured by NAC is accordingly that for an instance of VIM to succeed, NOP has to be non-accidentally satisfied.

The third and last necessary condition is:

CCC In order for a subject to construct a scenario $S_{i}$ involving an object $o$, the subject must consciously choose which properties $o$ is to have from a set of properties $\Pi$, which the subject has knowledge of. ${ }^{17}$

CCC's role in the argument is to make clear that it is the subject involved in VIM who actively chooses which properties to vary when passing from one scenario to the next.

Briefly stated, the problem Vaidya raises for the Husserlian approach with respect to these three conditions is the following: For VIM to reliably yield knowledge of essence, the subject applying the method must consider only variations which involve $o$ (by NOP). It furthermore needs to be the case that the relevant true belief generated by VIM is not brought about accidentally, i.e. in a manner conducive to an inadmissible case of epistemic luck (by NAC). The problem with the process then arises since the preservation of $o$ across the relevant scenarios is a matter of the preservation of $o$ 's essential properties. Since it is the subject involved in VIM which has to actively choose which properties to vary when imagining a new scenario (by CCC), what is needed to preclude the sort of

\footnotetext{
${ }^{15}$ Vaidya, "Understanding and Essence," 822.

${ }^{16}$ See e.g. Peter Unger, "An Analysis of Factual Knowledge," Journal of Philosophy 65, 6 (1968): 157-170, Duncan Pritchard, Epistemic Luck (Oxford: Oxford University Press, 2005). The notion will be discussed in a bit more detail later in the paper.

${ }^{17}$ Vaidya, "Understanding and Essence," 822.
} 


\section{Robert Michels}

accidentiality which is ruled out by NAC is a guarantee that the subject will vary only o's accidental properties when going through an instance of VIM, leaving its essential properties unchanged.

Vaidya's point is that such a guarantee could only be given if the subject knew beforehand which properties these were. We can try to make this more precise by spelling out the non-accidentiality requirement imposed by NAC in terms of a set of relevant possible worlds: For NAC to be satisfied, it has to be the case that in every relevant possible world in which the subject creates a scenario, the scenario is created by subtracting a non-essential property of $o$, resulting in a scenario centred on $o$ and not on some distinct object $o$ ' which differs from $o$ in its essential properties. Since according to CCC, the subject creates the scenario by conscious choice, this choice must be backed by a belief that the subtracted property is non-essential to $o$. Assuming that this backing-belief is present in all relevant relevant possible worlds, it follows that this belief of the subject satisfies a modal anti-luck luck condition for knowledge. While this does not logically entail that the subject knows that the relevant property is non-essential to $o$, anti-luck conditions are after all necessary, not sufficient for knowledge, we can, due to a lack of plausible alternative explanations, abductively infer that the subject has to have this piece of knowledge about $o$ 's essence. Granting this inference, we can conclude that the subject has to have knowledge of the essence of $o$ in order to gain knowledge of the essence of $o$ via VIM, resulting in a vicious epistemic circle. ${ }^{18}$ The, in my opinion correct, conclusion of the negative part of Vaidya's argument is that Husserl's method of free variation is not a suitable means to gain knowledge of essence.

18 See Vaidya, "Understanding and Essence," 823 for a more detailed presentation of the argument. Note that similar points have been raised by many others authors, including e.g. Peter H. Spader, "Phenomenology and the Claiming of Essential Knowledge," Husserl Studies 11, 3 (1994): 179 and Peter Simons, "Experience and Judgment: Investigations in A Genealogy of Logic, by Edmund Husserl," Journal of the British Society of Phenomenology 7, 1 (1976): 61-65. A range of similar objections is also critically discussed in David Kasmier, "A Defense of Husserl's Method of Free Variation," in Epistemology, Archaeology, Ethics. Current Investigations of Husserl's Corpus, eds. Pol Vandevelde and Sebastian Luft (London: Continuum, 2010): 21-40. 
Husserlian Eidetic Variation and Objectual Understanding...

\section{Vaidya's Positive Proposal: VIM as a Means to Gain Objectual Understanding of Essence}

\subsection{Objectual Understanding of Essence}

Vaidya argues that VIM still has a use in the epistemology of essence: It may not deliver knowledge, but it can instead be used to attain objectual understanding of essences. Some epistemologists have argued that understanding (including both its objectual and other varieties) is at least as important a subject of epistemological investigation as is knowledge. ${ }^{19}$ Vaidya's positive proposal hence promises to give us a new epistemic foundation for the epistemology of essence and potentially also the epistemology of modality more generally. ${ }^{20}$

There is no universal agreement about what understanding is in the contemporary literature. Among the accounts on offer, Vaidya in particular relies on Kvanvig's. Crucially, this account is based on the idea that 'understanding is not a species of knowledge. ${ }^{21}$ According to Kvanvig's account, understanding does however share, with an important qualification which will be discussed shortly, an important property of knowledge, namely its factivity. There is a near consensus that knowledge is factive, i.e. that for every $p$, if someone knows that $p$, then $p .^{22}$ While the consensus about the factivity of understanding, i.e. about whether the beliefs which an epistemic agent holds when understanding a subject have to be true, is not as strong as in case of knowledge, that some form of factivity holds is still the standard view. ${ }^{23}$ Kvanvig in particular points out that while 'understanding' is sometimes used non-factively in order to hedge claims which appear too strong, as e.g. in "My understanding is that you weren't home till after midnight," he still holds that the notion of understanding which is of interest to epistemologists is factive. ${ }^{24}$

Kvanvig accepts that propositional understanding, i.e. the sort of understanding at issue e.g. in "Claude understands that his internet connection is

${ }^{19}$ See Stephen R. Grimm, “The Value of Understanding," Philosophy Compass 7, 2 (2012): 103117.

${ }^{20}$ See Vaidya, "Understanding and Essence," 310-312.

${ }^{21}$ Jonathan L. Kvanvig, The Value of Knowledge and the Pursuit of Understanding (Cambridge: Cambridge University Press, 2003), 196.

22 See however Allan Hazlett, "The Myth of Factive Verbs," Philosophy and Phenomenological Research 80, 3 (2010): 497-522.

${ }^{23}$ Some non-factive cases of understanding are e.g. admitted in Linda Zagzebski, "Recovering Understanding," Knowledge, Truth, and Duty: Essays on Epistemic Justification, Responsibility, and Virtue, ed. Matthias Steup (Oxford: Oxford University Press, 2001).

${ }^{24}$ See Kvanvig, Value of Knowledge, 191. 
Robert Michels

slow," is factive. However, he explicitly relaxes this requirement with respect to objectual understanding, understanding of a more complex subject matter, e.g. as in "Claude understands quantum mechanics.": If someone has some false beliefs about a subject matter, then "we can ascribe understanding based on the rest of the information grasped that is true and contains no falsehoods," 25 as long as these "false beliefs concern matters that are peripheral rather than central to the subject matter in question." 26 Since factivity will play a crucial role in the argument of the next section, I will come back to this point.

Besides the similarity concerning factivity, there are two crucial differences between understanding and knowledge according to Kvanvig's account. First, "understanding requires, and knowledge does not, an internal grasping or appreciation of how the various elements in a body of information are related to each other in terms of explanatory, logical, probabilistic, and other kinds of relations that coherentists have thought constitutive of justification." 27 To illustrate this, consider Zeno, who has absolutely no idea about quantum mechanics, but has adopted a large set of true beliefs about the theory, including e.g. that the development over time of a quantum system is governed by Schrödinger's equation, because his physicist friend told him. Zeno can correctly be described as knowing the corresponding facts about quantum mechanics, even though it is clear that he completely lacks the grasping of the intrinsic structure of quantum mechanics which would be required for him to understand quantum mechanics.

Second, unlike knowledge, understanding is compatible with epistemic luck. ${ }^{28}$ Imagine for example that most books about politics were factually inaccurate, safe for the one excellent book which Xenia picked up randomly to learn about politics. The true beliefs about politics which Xenia acquires by reading the book would not constitute knowledge, since they are a product of epistemic luck. Her beliefs are, so to say, not modally stable enough to qualify as knowledge: She was very lucky to have picked the one factually accurate book and could easily have picked one of the many factually inaccurate ones instead. However, in the same scenario, Xenia could still acquire objectual understanding of politics. The presence of epistemic luck would not diminish Xenia's cognitive achievement of having developed an understanding of politics which manifests itself through her true beliefs about this subject matter.

\footnotetext{
${ }^{25}$ Kvanvig, Value of Knowledge, 201.

${ }^{26}$ Ibid.

${ }^{27}$ Kvanvig, Value of Knowledge, 192-3.

${ }^{28}$ See Kvanvig, Value of Knowledge, 199.
} 


\subsection{Vaidya’s Objectual Understanding-Based Proposal}

It is the second difference to knowledge which holds the key to Vaidya's positive proposal, i.e. its compatibility with epistemic luck. Vaidya's idea is to drop both NAC and NOP as necessary conditions on the success of VIM in order to repurpose it as a method for gaining objectual understanding of essence. Dropping NAC ensures that the epistemic circularity which affects the method in the knowledgecase no longer arises: The requirement of a guarantee that NOP is noncontingently satisfied drops out, which means that we no longer need to assume that a subject has to already have knowledge of the relevant essence.

If the epistemic circularity is already taken care of, why also drop NOP? Vaidya gives two reasons: First, the objectual understanding-based version of VIM may involve scenarios not centred on the object whose essence the subject aims to discover. According to Vaidya, such scenarios can contribute to the success of VIM since they allow the subject to "see how changing a property destroys the object" and "to comprehend why the property in question is essential."29

Second, Vaidya argues that NOP is an aboutness condition, which means that it ensures that VIM produces objectual understanding of the essence of the right object. This makes NOP an implicit second anti-luck condition, since it ensures that the true beliefs which a subject gains through VIM about $o$ 's essence are not merely by accident about that object, rather than another one. ${ }^{30}$

This second argument for rejecting NOP is, to my mind, unconvincing. NAC basically says that NOP has to be satisfied non-contingently, so if NOP itself already has a non-contingency condition built in, why doesn't this render NAC redundant? I will not go deeper into this question here for the simple reason that the argument of the next section will settle the status of NOP within Vaidya's proposal.

With the epistemic circularity taken care of, the following picture emerges: VIM is a method for acquiring objectual understanding of essence. Objectual understanding of essence is a state which i) involves true beliefs about essence, as it is factive, ii) is not subject to a version of NAC, as it is compatible with epistemic luck in acquiring these true beliefs, and iii) consists in a cognitively internal grasping of certain relations holding between different aspects of the essence of the object. ${ }^{31}$ Having described Vaidya's positive proposal for an epistemology of essence, I will now criticize it.

29 Vaidya, "Understanding and Essence," 824.

${ }^{30}$ See Vaidya, "Understanding and Essence," 826.

31 There are several questions about this proposal which one may ask. It does for example not address the metaphysical question of what the object of understanding is when one objectually 
Robert Michels

\section{Another Epistemic Circularity}

Vaidya's positive proposal includes the idea that scenarios which violate NOP can, and do, play a role in instances of VIM which lead to the acquisition of objectual understanding of essence. Here is how Vaidya explains their contribution:

Any scenario that does not contain $o$ is a scenario that plays a role in the subject arriving at an objectual understanding of the essence of $o$. In a scenario where some $o^{*}$ [i.e. an object distinct from $o$ ] is present the subject can see how changing a property destroys the object. This accidental insight into the object being changed allows the subject to comprehend why the property in question is essential. ${ }^{32}$

The general idea captured by this quote is that NOP-violating scenarios can contribute to one's gaining objectual understanding of essence by allowing one to see under which conditions the relevant object ceases to be itself. My focus for now will be on instances of VIM which involve such scenarios.

It is clear from Vaidya's negative argument that in a scenario of this sort, the subject would have to subtract one of the object $o$ 's essential properties. If it tried for example to gain objectual understanding of Socrates's essence, it might, let us assume, do this by subtracting his property of being human. With this kept in mind, let us come back to Vaidya's claim that a NOP-violating scenario can contribute to the success of an instance of VIM by allowing the subject to see that $o$ has ceased to be itself. For the point I am about to make it is important that objectual understanding is factive with respect to the relevant beliefs, i.e. in this case the belief that Socrates is essentially human. As I have pointed out in the previous section, Kvanvig's account of objectual understanding allows for someone to objectually understand a subject matter, even if they have some false beliefs about it, as long as these false beliefs concern only matters peripheral to the subject. Since the following argument requires factivity, I will now discuss to which extent this assumption can be made in the case of objectual understanding of essence, focusing in particular on whether Kvanvig's exception for peripheral beliefs applies in this context.

understands an essence. Kvanvig and others often talk of a subject or subject matter as the object of objectual understanding (see e.g. Kvanvig, Value of Knowledge, 197). This view for instance does not seem to square well with the traditional characterization of an object's essence in terms of its essential properties since it is unclear what the subject matter could be in this case. Further questions arise e.g. about the relation between the notion of judgment Vaidya relies on and those of belief and knowledge.

${ }^{32}$ Vaidya, "Understanding and Essence," 824. 


\section{Husserlian Eidetic Variation and Objectual Understanding...}

Granting Kvanvig's exception, which peripheral matters could a subject have false beliefs about without thereby undermining that it objectually understands an entity's essence? Since the essence of a subject matter concerns just those of its features which it has to have for it to be that subject matter, ${ }^{33}$ it is hard to see which beliefs about an entity's essence could count as peripheral, rather than central to it. It seems that the only plausible way to classify some beliefs about an entity's essence as peripheral is to accept Fine's distinctions between constitutive/consequential essence (intuitively, those parts of an entity's essence which are directly definitive of it/those parts which aren't, but rather are had essentially in virtue of other parts of its essence) and immediate/mediate essence (intuitively, the mediate essence of an entity also contains the essences of all entities on which it ontologically depends, whereas its immediate essence doesn't) and to identify the peripheral part of its essence with the union of its consequential and mediate parts. ${ }^{34}$ According to this way of drawing the distinction, at least the intersection of the immediate and constitutive parts of an entity's essence are central in Kvanvig's sense, which means that a subject must have true beliefs about them in order to objectively understand the entity's essence. Accordingly, understanding of immediate constitutive essence is indeed factive. In the following, my focus will first be exclusively on such beliefs, i.e. on beliefs about the immediate constitutive essence of entities. I will argue that an epistemic circularity similar to the one exploited by Vaidya in his negative argument will prevent subjects from acquiring understanding of immediate constitutive essence. After making this point, I will argue that this conclusion generalizes to essence in general. For the sake of simplicity, I will use 'essence' as a synonym for 'immediate constitutive essence' throughout the rest of this section, unless explicitly specified otherwise.

33 As per Aristotle's original characterization of essence as the 'what it is to be'. See his Metaphysics Z.4 and all contemporary accounts departing from it, including Fine's, Correia's, and Lowe's.

${ }^{34}$ See Fine, "Senses of Essence," sections 3 and 5 for the distinctions and see Kathrin Koslicki, "Varieties of Ontological Dependence," Metaphysical Grounding: Understanding the Structure of Reality, eds. Fabrice Correia and Benjamin Schnieder (Cambridge: Cambridge University Press, 2012), 190-195, Eileen S. Nutting and Ben Caplan and Chris Tillman, "Constitutive Essence and Partial Grounding," Inquiry 61, 2 (2018): 137-161, and Justin Zylstra, "Constitutive and Consequentialist Essence," Thought 8, 3 (2019): 190-199 for discussions of the first of the two distinctions. Note that this identification requires that the consequential parts of the relevant essence are merely consequential, i.e. not also constitutive and that it may be inadmissible for entities whose constitutive essence is essentially inferential, such as e.g. logical concepts like conjunction. 
Robert Michels

Let us for the sake of simplicity assume that a subject is going through an instance of VIM involving only one NOP-violating scenario, a scenario in which Socrates is not human and let us furthermore assume that it is part of Socrates's essence that he is human. The crucial point concerning this scenario for which I am going to argue now is that since only true beliefs about the essence of Socrates can constitute objectual understanding, the subject would have to recognize that this scenario does not involve Socrates in order to arrive at a (true) belief which can constitute understanding of Socrates's essence.

To see this point consider what would happen if it did not. In that case, some scenario or some scenarios produced by a subject going through VIM would not involve Socrates, but instead a non-human who resembles him in other respects. Now given the following success condition on VIM formulated by Husserl, it would follow that the subject acquires a false belief about Socrates's essence: To gain an insight into the essence of an object via an instance of VIM, the subject needs to grasp, as Husserl writes, the multiplicity of all the scenarios it has produced. Only by doing that can the subject isolate those features which stay constant throughout all imagined variations of the object and so ultimately gain insight into the object's essence. ${ }^{35}$ The condition requires the subject to grasp all the scenarios which of course includes any scenario(s) involving the non-human Socrates-like creature. The presence of at least one such scenario would therefore produce a false belief about the essence of Socrates in the subject. To generalize from the example, any instance of VIM which involves a NOP-violating scenario will produce a false belief in the subject.

Since objectual understanding of essence is factive, a subject cannot acquire objectual understanding of an object's essence via an instance of VIM if that instance involves at least one NOP-violating scenario. Based on this point, we can, just as in Vadiya's negative argument, abductively infer that a subject would have to recognize the NOP-violating scenarios involved in an instance of VIM as being such in order to avoid acquiring a false belief about the essence of an entity to finally gain understanding of it. The justification for this abductive inference, just as in Vaidya's argument, is that there is no other plausible explanation for what could enable a subject to successfully apply VIM to acquire understanding of the essence of an entity.

${ }^{35}$ As Husserl puts it, "[o]nly if we retain in grasp the things imagined earlier, as a multiplicity in an open process, and only if we look toward the congruent and the purely identical, do we attain the eidos [i.e. the ideal form which constitutes the essence]" (Husserl, Experience and Judgment," 343). 
Husserlian Eidetic Variation and Objectual Understanding...

So we face an epistemic circularity again: Only a subject which already has knowledge of the essence of the relevant object, or at least holds true beliefs about the essence of the relevant object as part of its understanding of that essence, can recognize that it has produced a NOP-violating scenario. Without this recognition on the side of the subject, the relevant instance of VIM will lead it to acquire false beliefs about the essence of the relevant object, leading to a violation of the factivity requirement. Hence, the subject could only successfully gain understanding of the essence of an object via a NOP-violating instance of VIM, if it already had the true beliefs it is supposed to acquire to gain this understanding. This shows that pace Vaidya, NOP-violating scenarios cannot contribute to the success of applications of VIM.

Now let us lift the restriction to immediate constitutive essence which was upheld throughout the preceding paragraphs. If NOP-violating instances of VIM do not allow a subject to acquire objectual understanding of immediate constitutive essence, what does this tell us about objectual understanding of essence in general? Recall that according to Kvanvig, someone can objectually understand a subject even though they hold false beliefs about the subject, as long as those false beliefs concern only peripheral matters, but not matters central to the subject. ${ }^{36}$ Given the plausible assumption that the matters which are central to an entity's essence are the immediate constitutive parts of its essence, this entails that a subject cannot objectually understand an entity's essence at all if it holds false beliefs about that essence's immediate constitutive parts. So for a subject to gain any sort of objectual understanding of essence, it has to have only true beliefs about the constitutive immediate parts of this essence. Since I have just (abductively) argued that subjects cannot plausibly satisfy this condition in the context of a NOP-violating instance of VIM, the conclusion that instances of VIM which involve NOP-violating scenarios do not allow them to acquire objectual understanding of essence generalizes to essence tout court, i.e. the broader notion of essence which also includes consequential and mediate essence.

An immediate consequence of the conclusion of the preceding argument is that a subject can only successfully acquire objectual understanding of an object's essence via an instance of VIM, if this instance involves no NOP-violating scenarios. But this just means that, pace Vaidya, NOP has to be part of the positive proposal. Does re-introducing this principle finally give us a workable understanding-based epistemology of essence?

${ }^{36}$ See again Kvanvig, Value of Knowledge, 201. 
Robert Michels

\section{Can Vaidya's Proposal be Fixed By Re-Introducing NOP?}

Can one simply reintroduce NOP as a component of Vaidya's proposal? If Vaidya is right to think of NOP as an anti-luck condition, then one cannot consistently do so: Objectual understanding is by definition compatible with epistemic luck, so an epistemology of essence centred on this notion cannot involve an anti-luck condition. There are two ways around this problem: Pace Vaidya, one might just deny that NOP is an anti-luck condition. This would immediately dissolve the problem and NOP could consistently be retained as a condition on the success of VIM.

Second, even if one granted Vaidya's claim about his version of NOP, one might avoid the problem by re-introducing a de-modalizing version of the principle which eliminates the modal auxiliary verb 'must:'

NOP* An instance of VIM only yields a judgment about whether $P$ is an essential property of $o$ through property variation on $o$ via the construction of a set of scenarios $S_{1 \ldots} . . S_{n}$, if it is the case that $o$ is preserved in the transition from each $S_{i}$ to $S_{k}$.

Since NOP* is a non-modal principle, assuming that the conditional involved is the material conditional, it cannot be considered an anti-luck condition, neither in the sense of a safety, nor in that of a sensitivity condition. ${ }^{37}$ Still, it can block the circularity problem arising from NOP-violating scenarios. Just like NOP, NOP* makes the preservation of $o$ throughout all scenarios involved in an instance of VIM a necessary condition for its success. The new principle could therefore still do its job and it could furthermore consistently be added as a success condition on Vaidya's version of VIM.

Re-introducing NOP or NOP* indeed saves Vaidya's proposal from the problem raised in the previous section. But does this addition result in a workable variant of Vaidya's proposal? There are three important reasons to doubt this.

\footnotetext{
${ }^{37}$ Anti-luck conditions in epistemology are usually spelled out in terms of a range of closest possible worlds and are therefore essentially modal: Safety conditions require that for a subject to know that $p, p$ has to be true in the closest worlds in which it is believed (see e.g. Ernest Sosa, "How to Defeat Opposition to Moore," Philosophical Perspectives 13 (1999): 137-49, Pritchard, Epistemic Luck, ch. 6, Timothy Williamson, Knowledge and its Limits (Oxford: Oxford University Press, 2000), chs. 4-5, sensitivity conditions in contrast require that $p$ is not believed by the subject in the closest non- $p$ worlds (see e.g. Robert Nozick, Philosophical Explanations (Cambridge: The Belknap Press of Harvard University Press, 1981): ch. 3.
} 
Husserlian Eidetic Variation and Objectual Understanding...

5.1 VIM's Quality as a Method for Acquiring Objectual Understanding of Essence

It is clear that Husserl considers VIM to be a method. To be more specific, a method "for the acquisition of pure concepts or concepts of essences." 38 Surprisingly, Vaidya never refer to VIM as a method in his "Understanding and Essence." Yet, he never explicitly denies that it is one either. Since it is very hard to see what else VIM could be, I will follow Husserl in assuming that VIM is a method.

The question which I will discuss in this subsection is whether VIM is a good method, a method which a subject seeking to acquire objectual understanding of essence could rationally adopt to pursue this goal. There are many different criteria for the overall quality of an epistemological method. I will here focus on two important quality criteria: First, the method's rate of success and second, what I will call its transparency. I call a method transparent, if it affords the subject who applies it feedback on whether it can terminate with success during different stages of its application. The main point of this subsection will be that VIM fares badly with respect to both of these criteria. My argument for this point will rely on a systematic connection between the two criteria.

Let me start with VIM's transparency. A point made in the previous section was that a subject going through an instance of VIM cannot recognize a NOPviolating scenario as such. To do so, it would have to be able to recognize this scenario as resulting from the subtraction of an essential property of the object, because this is the only plausible way to guarantee that an application of VIM involving such a scenario could succeed. This, the subject cannot do, because this would require it to already have the sort of robust epistemic access to the essence of the relevant object which it is seeking to acquire by applying VIM. This point can be generalized to NOP-conform scenarios: In order to recognize a scenario as such, the subject would have to recognize that the property which it subtracted to create the scenario was not an essential property of the relevant object. This would also require the subject to already have prior epistemic access to the relevant object's essence which, by hypothesis, it does not have. So a subject who relies on VIM to acquire objectual understanding of essence can, on pain of epistemic circularity, neither recognize the scenarios which it creates while going through this process as NOP-violating, nor as NOP-conform.

NOP spells out a success-condition for VIM, a condition which has to be met for an instance of VIM to succeed. Since a subject cannot recognize of any scenario whether it conforms or fails to conform to this success condition, a subject

38. Husserl, Experience and Judgment, 340. 
Robert Michels

applying the method cannot, at any stage of the process, tell whether its application of VIM meets this success-condition.

This generalizes an important point about the nature of Vaidya's proposal, which he explicitly mentions: Even if a subject manages to acquire objectual understanding of an object's essence through VIM, this epistemic state does not involve the subject's recognition that it has in fact attained this state. This follows directly from the account of objectual understanding which Vaidya accepts. ${ }^{39}$ The point just made shows that this lack of recognition not only affects a subject after it has successfully applied VIM, but rather it completely pervades any instance of VIM at all of its stages, from the very first scenario on. We can conclude that VIM is lacking in transparency and fares badly with respect to this first qualitycriterion.

Let me now focus on VIM's rate of success. Concerning this factor, I have a speculative argument to offer. Consider the class of all instances of VIM. Can we say anything about how many of these instances may succeed? To be sure, we cannot give a precise answer since we can neither determine the total number, nor the number of the successful instances of VIM. There are however two things we know: First, only NOP-/NOP*-conform instances can succeed, and second, VIM is intransparent in that a subject going through an instance of the process cannot, at any stage, tell whether it conforms to NOP. These two point taken together with a third strongly suggest that VIM does not have a good rate of success. The third point is that following Husserl, VIM is designed to have "a structure of arbitrariness," 40 which means that the process of producing scenarios in an instance of VIM, or to use Husserl's terminology, variants in an application of the method of essential seeing, is such that "it is a matter of indifference what might still be joined to it, a matter of indifference what, in addition, I might be given to apprehend in the consciousness that 'I could continue in this way." 41 The underlying idea is of course that an object's essence will stay the same throughout all possible scenarios, no matter how arbitrary the changes are which the subject makes to produce them.

If we put this third point and the first two together, we get that VIM is a process which is very likely to fail: The process is designed to allow the subject to create scenarios by arbitrarily varying the properties of the object on which the scenarios are centred and it can only successfully terminate if these scenarios all conform to NOP. However, the subject cannot recognize whether the scenarios it

\footnotetext{
${ }^{39}$ See Vaidya, "Understanding and Essence," 827-828.

${ }^{40}$ Husserl, Experience and Judgment, 342.

${ }^{41}$ Ibid.
} 
Husserlian Eidetic Variation and Objectual Understanding...

creates conform to this success condition or whether they don't. The success of an application of VIM therefore appears to be mostly a matter of luck, since the subject has to create scenarios without any means to verify that they do not undermine the successful completion of the process. This does not show that VIM cannot succeed, but it strongly suggests that it is, taking into account two plausible quality criteria, not a good method for acquiring objectual understanding of essence.

\subsection{The Contemporary View of Essence and the Apriority of VIM}

Vaidya aims to develop a general epistemology of essence which fits the contemporary view of essence of philosophers such as Fine and Lowe. ${ }^{42}$ Following Kripke, ${ }^{43}$ this view characteristically admits cases in which the essence of an entity is not discoverable by a priori means: The microstructure of a substance might for example be said to be essential to it, even though it is not discoverable by a priori means. More generally, the idea is that the two distinctions of it being imaginable or not that an object has a certain property and of it being essential or accidental to that object whether it has that property cut across each other. VIM faces a fundamental problem in accounting for knowledge of/understanding of an entity's essence if we assume this contemporary view of essence.

When describing the method of essential seeing, Husserl is clear that he assumes there to be a close connection between the thinkable and imaginable and the essential: "The essence proves to be that without which an object of a particular kind cannot be thought, i.e., without which the object cannot be intuitively imagined as such." 44 According to Husserl, "[w]e can direct our regard toward it[the essence] as toward the necessarily invariable, which prescribes limits to all variation practiced in the mode of the "arbitrary." 45 With the contemporary notion of essence in mind, this quotation could be taken to give us a possible answer to our problem since one might understand it to say that while engaging in the imaginative activity required by VIM, the subject's imagination is limited by the relevant essence. ${ }^{46}$ This limitation would then be what prevents the subject

42 See Vaidya, "Understanding and Essence," 819.

${ }^{43}$ See Kripke, Naming and Necessity.

${ }^{44}$ Husserl, Experience and Judgment, 341. My emphasis.

${ }^{45}$ Ibid.

46 Note that this contradicts the interpretation of Husserl developed in De Santis, "Phenomenological Kaleidoscope," 31-33. Since my concern here is not with Husserl's own view of the method, but rather with a view which adapts it to the notion of essence of contemporary metaphysicians, I will only remark that this interpretation would not help in solving the 


\section{Robert Michels}

from producing a variation in which the relevant object lacks one of its essential properties. One might hence say that there is a pre-established harmony between what we can imagine about an object of a certain kind and its corresponding kindessence. If we import this idea into the contemporary context, it amounts to what one may call essential rationalism: ${ }^{47}$ A philosopher who accepts it assumes that the imagination-based method of essential seeing gives us a priori access to essence.

What VIM could hence offer a contemporary epistemologist of essence is a systematic method for probing which of an object's properties we can subtract from it in our imagination and which we cannot, presupposing the essential rationalist view that what we can imagine about an object coincides with the possible states of the object left open by its essence. This presupposition however directly conflicts with the contemporary view that imaginability and essentiality come apart. The question of how to bridge this gap, or the corresponding gap between conceivability and metaphysical possibility, is of course one of the core questions in contemporary epistemology of modality. ${ }^{48}$ An answer to this question would be vital to Vaidya's proposal and more generally to any epistemology of essence based on the Husserlian method of essential seeing. It is not surprising that Husserl himself ${ }^{49}$ provides no answer to this contemporary question, but we do not find it addressed in Vaidya's paper either. What is clear however is that from a contemporary perspective, the view about the relation between the imaginable and the essential which one can extract from Husserl falls short of providing a satisfying answer.

\subsection{Conflation of Essence and de re Modality}

A further problem with the modified version of Vaidya's proposal concerns the relation between the notions of essence and of metaphysical necessity, two notions which are clearly distinguished by contemporary essentialists. ${ }^{50}$

Consider an instance of VIM. In it, the subject starts out with a particular experience or intuition of an object which is then subsequently modified by

problem at hand since it takes the subject who applies the method itself, not the relevant essence, to be the source of this limitation.

${ }^{47}$ Compare modal rationalism as e.g. characterized in Chalmers, "Does Conceivability Entail Possibility?" 172-173.

${ }^{48}$ See e.g. Chalmers, "Does Conceivability Entail Possibility?" and Peter Kung, "Imagining as a Guide to Possibility," Philosophy and Phenomenological Research 81, 3 (2010): 620-663.

49 Who holds that "essential truths are called a priori; this means, by reason of their validity, preceding all factuality, all determinations arising from experience." (Husserl, Experience and Judgment, 352-353).

${ }^{50}$ I owe the main idea for this objection to Claudio Calosi and Fabrice Correia. 
Husserlian Eidetic Variation and Objectual Understanding...

subtracting properties of the object to produce the plurality of variations which is then considered as a whole by the subject in order to see which properties remain constant throughout all of them. Both the subject's starting point and the variations produced can be represented, without giving up on anything which is essential to the intended workings of VIM, in terms of either sets of propositions or facts involving the relevant object. Since possible worlds correspond to maximally consistent sets of propositions or facts, this means that we can think of them as corresponding to parts of possible worlds. This should immediately give us pause.

Contemporary essentialists hold that essence is not definable in terms of necessity. The notion of metaphysical necessity (de re) can adequately be captured using possible worlds, but the notion of essence cannot be: Possible worlds are an adequate tool for capturing the metaphysically necessary properties of an object, but they cannot be used to capture the object's essence, since there are some properties which some objects have with metaphysical necessity, even though they do not belong to these object's essences: Socrates is necessarily distinct from the Eiffel Tower, but it is not essential to him that he is. This is of course the core insight of Fine's influential objections to the modal definition of essence. ${ }^{51}$

Since VIM can equivalently be restated as a method which relies only on possible worlds, this crucial difference means that there is in fact no good reason to think that the method can allow us to acquire (knowledge or) understanding of the essential, rather than of the necessary properties of an object. To put the point differently, from a contemporary perspective, essence is a hyperintensional notion, but Vaidya's variant of the Husserlian method is only intensional in nature. What we are looking for is a foundational method for the epistemology of essence, but it turns out that we have no good reason to think that VIM gives us such a method, as opposed to a method for acquiring (knowledge or) understanding of metaphysical necessity (de re).

\section{Whither Essential Seeing and Objectual Understanding of Essence?}

Vaidya's proposal combines two components which have been largely neglected in the contemporary discussions about the epistemologies of essence and of modality. The first is the Husserlian method of essential seeing, the second the idea of refocusing the debate on understanding instead of knowledge. The focus so far was on the combination of the two, but in this last section, I want to briefly comment on them separately in light of the previous discussion, starting with the Husserlian method/VIM.

${ }^{51}$ See Fine, "Essence and Modality." 
Robert Michels

It is important to note that the problems for VIM which were raised in the previous section arise no matter whether the method aims at understanding or at knowledge. Since I do not see any salient alternatives to these two proposals, I believe that VIM cannot play the central role in the epistemology of essence which Vaidya allocates to it. This role requires a method which permits a subject to acquire arbitrary bits of objectual understanding (or knowledge) of essence without having any previous epistemic access to essence, something which VIM does not deliver.

That said, there still are two roles which it might be able to play. First, VIM appears to give us a viable method for determining, within certain limits, the essential properties of objects which belong to domains which are wholly accessible via our imagination and more generally via a priori methods. This may be the case for some geometrical objects, as Tieszen argues. ${ }^{52}$

Second, VIM may give us a general systematic method for making tacit knowledge of essence explicit. ${ }^{53}$ It is plausible that freely varying the properties of an object may help us get clear on which of an object's properties we assume to essentially belong to it. Assuming that we can acquire knowledge or understanding of essence in another way, VIM may thus help us realize which of our knowledge about an object concerns its essence. Another way in which the method may help us in this manner is as a device for inductively generalizing from knowledge or understanding of individual essences. A subject might for example apply VIM to different objects which share a certain essential property, noticing in each case that another property of these objects can likewise not coherently be subtracted when forming a new scenario. Based on this realization, the subject may inductively (and therefore defeasibly) infer that the two relevant properties are essentially connected with each other. This in turn may allow the subject to form the hypothesis that the second property is also essential to objects which have the first.

Can Vaidya's proposal to refocus the epistemology of essence on objectual understanding help solve problems faced by a knowledge-based epistemology? As I have stressed in the previous section, to have objectual understanding of an object's essence, a subject still needs to have true beliefs about its essence. In this respect, any epistemology of essence which builds on the former instead of the latter notion will still have to answer the question of how we can reliably acquire true

52 See Richard Tieszen, "Free Variation and the Intuition of Geometric Essences: Some Reflections on Phenomenology and Modern Geometry," Philosophy and Phenomenological Research 70, 1 (2005): 153-173.

53 Thanks to Olivier Massin for this suggestion. An interpretation of Husserl's own method which points in this direction is proposed in Kasmiers, "Defense of Husserl's Method." 
Husserlian Eidetic Variation and Objectual Understanding...

beliefs about essence. So it seems that the move to objectual understanding does not really help us address the most pressing fundamental question in the epistemology of essence. This problem is magnified by the fact that objectual understanding, as Kvanvig and Vaidya understand it, is by design unsuitable to give us a reliable method, since it is compatible with epistemic luck. Perhaps other conceptions of objectual understanding could fare better in this respect, but this is a topic for another day. ${ }^{54}$

${ }^{54}$ I would like to thank participants of sessions of the research colloquium at the Institute de philosophie of the University of Neuchâtel and of the eidos seminar at the University of Geneva. Special thanks to Claudio Calosi, Donnchadh O'Connail, Fabrice Correia, Arturs Logins, Olivier Massin, Kevin Mulligan, Maria Scarpati, Peter Simons, Jonas Waechter and three anonymous reviewers. The first steps towards writing this paper were made several years ago in a section of my phd thesis for which I received funding from the European Community's Seventh Framework Programme FP7/2007-2013 under grant agreement no. FP7-238128. Further work on the paper was made possible by the SNSF-funded project 'Identity in Cognitive Science, Quantum Mechanics, and Metaphysics,' grant number 185435, Università della Svizzera italiana, PI: Kevin Mulligan. 